Original Article

\title{
SYNTHESIS, IN VITRO ANTIMICROBIAL ACTIVITY OF SCHIFF'S BASE, AZETIDINONES AND THIAZOLIDINONES
}

\author{
BHAVNABEN D. MISTRY*, KISHOR R. DESAI**, NIGAM J. DESAI** \\ *Department of Chemistry, B. K. M. Science College, Valsad, **Department of Chemistry, Uka Tarsadia University, Bardoli-Surat \\ Email: bhavana_mistry11@yahoo.co.in
}

Received: 01 Oct 2016, Revised and Accepted: 05 Dec 2016

\section{ABSTRACT}

Objective: The objective of the present study is to synthesize 3-Chloro-4-[3-(2,4-dichloro-5-fluoro phenyl)-1H-pyrazol-4-yl]-1-(substituted) azetidin-2-one [4a-n] and 2-[3-(2,4-Dichloro-5-fluoro phenyl)-1H-pyraol-4-yl]-3-(substituted phenyl)-1,3-thiazolidin-4-one [5a-n]. The structure of all synthesized compounds were characterized by IR, ${ }^{1} \mathrm{H}$ NMR, ${ }^{13} \mathrm{C}$ NMR and mass spectral studies.

Methods: The titled compounds 3-Chloro-4-[3-(2,4-dichloro-5-fluoro phenyl)-1H-pyrazol-4-yl]-1-(substituted) azetidin-2-one [4a-n] and 2-[3-(2,4Dichloro-5-fluoro phenyl)-1H-pyraol-4-yl]-3-(substituted phenyl)-1,3-thiazolidin-4-one [5a-n] were synthesized by the reaction of N-\{[3-(2,4-dichloro-5fluoro phenyl)-1H-pyraol-4-yl] methylene $\}$ substituted anilin [3a-n] with chloro acetyl chloride and thioglycolic acid respectively. Compounds $\mathrm{N}-\{[3-(2,4-$ dichloro-5-fluoro phenyl)-1H-pyraol-4-yl] methylene\} substituted aniline [3a-n] were synthesized by the reaction of 3-(2,4-dichloro-5-fluoro phenyl)-1Hpyrazol-4-carbaldehyde [2] with primary aromatic amine in alcohol. All compounds were evaluated for their antimicrobial activity.

Results: Compounds 3a,3b,3d,3j,3l,4d,4e,4j,4l,4m,5e,5g,5h,5n exhibited excellent to good antibacterial activity as compared to reference drugs.

Conclusion: In summary, N-\{[3-(2,4-dichloro-5-fluoro phenyl)-1H-pyraol-4-yl] methylene \} substituted anilin [3a-n], 3-Chloro-4-[3-(2,4-dichloro-5-fluoro phenyl)-1H-pyrazol-4-yl]-1-(substituted) azetidin-2-one [4a-n] and 2-[3-(2,4-Dichloro-5-fluoro phenyl)-1H-pyraol-4-yl]-3-(substituted phenyl)-1,3thiazolidin-4-one [5a-n] derivatives have been synthesized and characterized. In vitro antimicrobial testing of the compounds was carried out by microdilution Method. Amongst the synthesised compounds, many of them had proven their antimicrobial potency which varies from good to excellent.

Keywords: Schiff's base, 2-Azetidinone, 4-Thiazolidinone, Antimicrobial activity

(C) 2016 The Authors. Published by Innovare Academic Sciences Pvt Ltd. This is an open access article under the CC BY license (http://creativecommons.org/licenses/by/4.0/) DOI: http://dx.doi.org/10.22159/ijcpr.2017v9i1.16634

\section{INTRODUCTION}

Heteroaromatic compounds have attracted considerable attention in the design of biologically active molecules and advanced organic materials. Heterocycles containing nitrogen atoms in the core structure shows a number of pharmacologically and biologically active compounds. Hence, a practical method for the preparation of such compounds is of great interest in synthetic organic chemistry. Structurally, a Schiff's base (also known as imine or azomethine) is a nitrogen analogue of an aldehyde or ketone in which the carbonyl group has been replaced by an imine or azomethine group. Schiff's bases of pyrazole aldehydes and aromatic amines exhibit a wide range of biological activities such as antifungal [1], antibacterial [2] and antitubercular [3] etc. The biological significance of this class of compounds impelled us to continue working on the synthesis of new schiff's bases of pyrazole derivatives.

$\beta$-Lactam containing antibacterial agents has become an integral part of chemotherapeutic arsenal available to today's medical practitioners. Although the number of existing agents are quite extensive, but the search for better and more effective drug is still going on. Azetidinones are the very important class of compounds possessing a wide range of biological activities such as antibacterial [4], anti-inflammatory [5], antihyperlipidemic [6], anticancer [7], antimicrobial [8], antitumor [9], antitubercular [10] etc. Furthermore, thiazolidinone derivatives found to possess a wide spectrum of biological activities [11-17].

\section{MATERIALS AND METHODS}

Melting points were determined by open capillaries and are uncorrected. The progress of the reaction was checked on aluminium coated TLC plates (E. Merck) using various solvent systems as mobile phase and visualised under iodine vapour. IRspectra $\left(\mathrm{cm}^{-1}\right)$ were recorded on a Shimadzu FT-IR spectrophotometer using $\mathrm{KBr}$ pellet method. ${ }^{1} \mathrm{H}$ NMR and ${ }^{13} \mathrm{C}$ NMR spectra were recorded on a Bruker DRX-300 NMR instrument, using $\mathrm{CDCl}_{3}$ as solvent and TMS as an internal reference (chemical shifts in $\delta$, ppm). Mass spectra were obtained on an Agilent 6520 (Q-TOF) Mass spectrometer.

Synthesis of (1E)-1-(2,4-Dichloro-5-fluorophenyl) ethanone hydrazone [1]

A mixture of 2,4-dichloro-5-fluoro acetophenone $(0.01 \mathrm{~mol})$ and hydrazine hydrate $(0.012 \mathrm{~mol})$ was refluxed in round bottom flask containing absolute alcohol $(30 \mathrm{ml})$ for $2 \mathrm{~h}$ in the presence of few drops of acetic acid. The content of the flask was cooled to give a solid product which was filtered, washed with water, dried and recrystallized from ethanol as a yellow crystalline solid.

Synthesis of 3-(2,4-dichloro-5-fluoro phenyl)-1H-pyrazol-4carbaldehyde [2]

To a cold solution of (1E)-1-(2,4-Dichloro-5-fluoro phenyl)ethanone hydrazone $(0.015 \mathrm{~mol})$ in DMF $(25 \mathrm{ml})$ was added $\mathrm{POCl}_{3}(0.0395$ mol) and resulting mixture was stirred at $55-60{ }^{\circ} \mathrm{C}$ for $5-6 \mathrm{~h}$ [18]. Then the mixture was cooled to room temperature and poured into ice cold water.

A saturated solution of bicarbonate was added to neutralise the solution. The precipitate so formed was filtered, washed with water, dried and recrystallized from ethanol as a yellowish white crystalline solid.

General procedure for the synthesis of N-\{[3-(2, 4-dichloro-5-fluoro phenyl)-1H-pyraol-4-yl] methylene\} substituted anilin [3a-n]

A mixture of 3-(2,4-dichloro-5-fluoro phenyl)-1H-pyrazol-4carbaldehyde $(0.01 \mathrm{~mol})$, various primary aromatic amine $(0.01$ mol) and few drops of gla. Acetic acid was refluxed in methanol for six hours. Then the refluxed content was cooled to room 
temperature and solid separated was filtered, washed with water and recrystallized from acetone.

[3d] IR (KBr cm$\left.{ }^{-1}\right): 3389.81(-\mathrm{NH}), 1567.08(\mathrm{C}=\mathrm{N}), 807.10(\mathrm{C}-\mathrm{Cl})$, 1094.22 (C-F), $1201.49(-\mathrm{C}-\mathrm{N}){ }^{1} \mathrm{H}_{\mathrm{NMR}}\left(\mathrm{CdCl}_{3}\right) \delta: 6.972(-\mathrm{NH}), 5.996$ $(-\mathrm{CH}), 7.361-7.572(\mathrm{Ar}-\mathrm{H}), 7.696$ (CH-Cl), 7.701 (CH-F), 9.747 ($\mathrm{CH}=\mathrm{N}) .{ }^{13} \mathrm{C}$ NMR: $161.38(\mathrm{C} 1), 120.09(\mathrm{C} 2), 129.69(\mathrm{C} 3), 129.08(\mathrm{C} 4)$, 139.48(C5), 115.4(C6), 139.85(C7), 110.05(C8), 133.45(C9), 160.00(C10), 150.02(C11), 122.99(C12), 125.85(C13), 131.88(C14), 125.85(C15), 122.99(C16). Mass (m/z): 368.5 (M), $374.5(\mathrm{M}+6), 257$ $230,205,164,138$.

[3m] IR ( $\left.\mathrm{KBr} \mathrm{cm}^{-1}\right): 1567.20(\mathrm{C}=\mathrm{N}), 807.32$ (C-Cl), 1095.34 (C-F), $1023.0\left(-\mathrm{OCH}_{3}\right), 1202.57(-\mathrm{C}-\mathrm{N}) .{ }^{1} \mathrm{H}$ NMR $\left(\mathrm{CdCl}_{3}\right) \delta: 6.996(-\mathrm{NH})$, 5.877 (-CH), 7.328-7.535 (Ar-H), 7.697 (CH-Cl), 7.702 (CH-F), 9.750 $(-\mathrm{CH}=\mathrm{N}), \quad 3.800 \quad\left(-\mathrm{OCH}_{3}\right) .{ }^{13} \mathrm{C}$ NMR: $161.45(\mathrm{C} 1), 119.99(\mathrm{C} 2)$, 129.78(C3), 129.59(C4), 139.45(C5), 114.44(C6), 139.95(C7), 110.05(C8), 134.55(C9), 159.97(C10), 139.45(C11), 123.25(C12), 122.25(C13), 128.08(C14), 115.50 (C15), 153.67(C16).

Mass (m/z): 364 (M), $368(M+4), 257,230,200,164,134$.

General procedure for the synthesis of 3-Chloro-4-[3-(2,4-dichloro-5fluoro phenyl)-1H-pyrazol-4-yl]-1-(substituted) azetidin-2-one [4a-n]

Compound N-\{[3-(2,4-dichloro-5-fluoro phenyl)-1H-pyrazol-4-yl] methylene $\}$ substituted anilin $(0.01 \mathrm{~mol})$ was dissolved in 1,4-dioxan $(50 \mathrm{ml})$. To this solution chloro acetyl chloride $(0.012 \mathrm{~mol})$ was added drop wise with constant stirring maintaining the temperature below $10{ }^{\circ} \mathrm{C}$ and then tri ethyl amine $(0.02 \mathrm{~mol})$ was added to it. The mixture was stirred for $2 \mathrm{~h}$. The reacrtion mixture was then refluxed for 9-10 h. The resulting solution was then poured into crushed ice and the product thus obtained was filtered, washed with water and recrystallized from ethyl acetate.

[4a] IR (KBr cm$\left.{ }^{-1}\right)$ : 1730 (C=0), 1203 (CH-N), 760 (C-Cl), 1062 (C-F). ${ }^{1} \mathrm{H}$ NMR $\left(\mathrm{CdCl}_{3}\right) \delta$ : 6.930 (-NH pyrazol), $5.958(-\mathrm{CH}$ pyrazol), $5.103(\mathrm{CH}-\mathrm{N})$, 3.885 (CH-Cl), 7.307-7.960 (Ar-H). ${ }^{13} \mathrm{C}$ NMR: 161.79(C1), 120.0(C2), 131.69(C3), 129.31C4), 138.60(C5), 117.17(C6), 143.12(C7), 114.3(C8), 133.18(C9), 60.5(C10), 62.0(C11), 162.08(C12), 140.75(C13), 119.77(C14), 130.69(C15), 123.05(C16), 129.31(C17), 117.17(C18). Mass (m/z): 409 (M), 415 (M+6), 333.5, 246.5, 230, 164, 141.5. [4j] IR ( $\mathrm{KBr} \mathrm{cm}^{-1}$ ): 1712 (C=0), 1205 (CH-N), 758 (C-Cl), 1070 (C-F), $2919\left(-\mathrm{CH}_{3}\right) .{ }^{1} \mathrm{H}$ NMR $\left(\mathrm{CdCl}_{3}\right) \delta: 6.931(-\mathrm{NH}$ pyrazol), $5.961(-\mathrm{CH}$ pyrazol), $5.105(\mathrm{CH}-\mathrm{N}), 3.940(\mathrm{CH}-\mathrm{Cl}), 7.305-8.049(\mathrm{Ar}-\mathrm{H}), 2.529(-$ $\left.\mathrm{CH}_{3}\right) .{ }^{13} \mathrm{C}$ NMR: $161.59(\mathrm{C} 1), 120.0(\mathrm{C} 2), 130.69(\mathrm{C} 3), 129.5(\mathrm{C} 4)$, 139.97(C5), 117.29(C6), 144.15(C7), 114.39(C8), 133.19(C9), 60.05(C10), 62.0(C11), 162.0(C12), 138.0(C13), 120.48(C14), 129.31(C15), 133.38(C16), 129.31(C17), 120.48(C18). Mass (m/z): 424.5 (M), 430.5 (M+6), 333.5, 307, 259, 196, 164.

General procedure for the synthesis of 2-[3-(2,4-Dichloro-5fluoro phenyl)-1H-pyraol-4-yl]-3-(substituted phenyl)-1,3thiazolidin-4-one [5a-n]

A mixture of N-\{[3-(2,4-dichloro-5-fluoro phenyl)-1H-pyrazol-4-yl] methylene substituted anilin $(0.01 \mathrm{~mol})$, thio glycolic acid $(0.01$ mol) and anhydrous zinc chloride $(0.01 \mathrm{~mol})$ in DMF was refluxed for 11-12 h. The resulting solution was then poured into crushed ice and the product thus obtained was filtered, washed with cold water and recrystallized from methanol.

[5a] IR ( $\left.\mathrm{KBr} \mathrm{cm}^{-1}\right): 1720.20$ (C=0), 1260.70 (CH-N), 760.26 (-Cl), $1066.12(-\mathrm{F})$.

${ }^{1} \mathrm{H} \mathrm{NMR}\left(\mathrm{CdCl}_{3}\right)$ ) 6.927 (-NH pyrazol), 5.959 (-CH pyrazol), 5.871 (CHN), $4.01\left(\mathrm{CH}_{2}-\mathrm{S}\right), 7.324-7.704(\mathrm{Ar}-\mathrm{H}) .{ }^{13} \mathrm{C}$ NMR: $161.41(\mathrm{C} 1), 119.99(\mathrm{C} 2)$ 131.91(C3), 129.70(C4), 139.82(C5), 117.18(C6), 144.65(C7), 108.74(C8), 135.82(C9), 66.1(C10), 34.0(C11), 171.1(C12), 141.75(C13), 126.4(C14), 131.82(C15), 129.30(C16), 131.82(C17), 126.4(C18). Mass (m/z): 408(M), $412(\mathrm{M}+4), 380,331,245,230,164$.

[5e] IR ( $\left.\mathrm{KBr} \mathrm{cm}^{-1}\right): 1733.35$ (C=0), $1256.46(\mathrm{CH}-\mathrm{N}), 766.17$ (-Cl), $1063.31(-\mathrm{F}), 1345.04\left(-\mathrm{NO}_{2}\right) .{ }^{1} \mathrm{H}$ NMR $\left(\mathrm{CdCl}_{3}\right) \quad \delta: 6.927 \quad(-\mathrm{NH}$ pyrazol), 5.959 (-CH pyrazol), $5.923(\mathrm{CH}-\mathrm{N}), 3.975\left(\mathrm{CH}_{2}-\mathrm{S}\right), 7.323-$ $7.703(\mathrm{Ar}-\mathrm{H}) .{ }^{13} \mathrm{C}$ NMR: $161.48(\mathrm{C} 1), \quad 119.99(\mathrm{C} 2), 131.91(\mathrm{C} 3)$, 129.67(C4), 140.05(C5), 117.18(C6), 144.75(C7), 108.74(C8), 135.82(C9), 66.16(C10), 42.36(C11), 171.2(C12), 131.82(C13), 108.74(C14), 131.91(C15), 119.76(C16), 126.44(C17), 141.75(C18). Mass (m/z): 453 (M), 457 (M+4), 421, 331, 289, 230, 212, 164, 122.

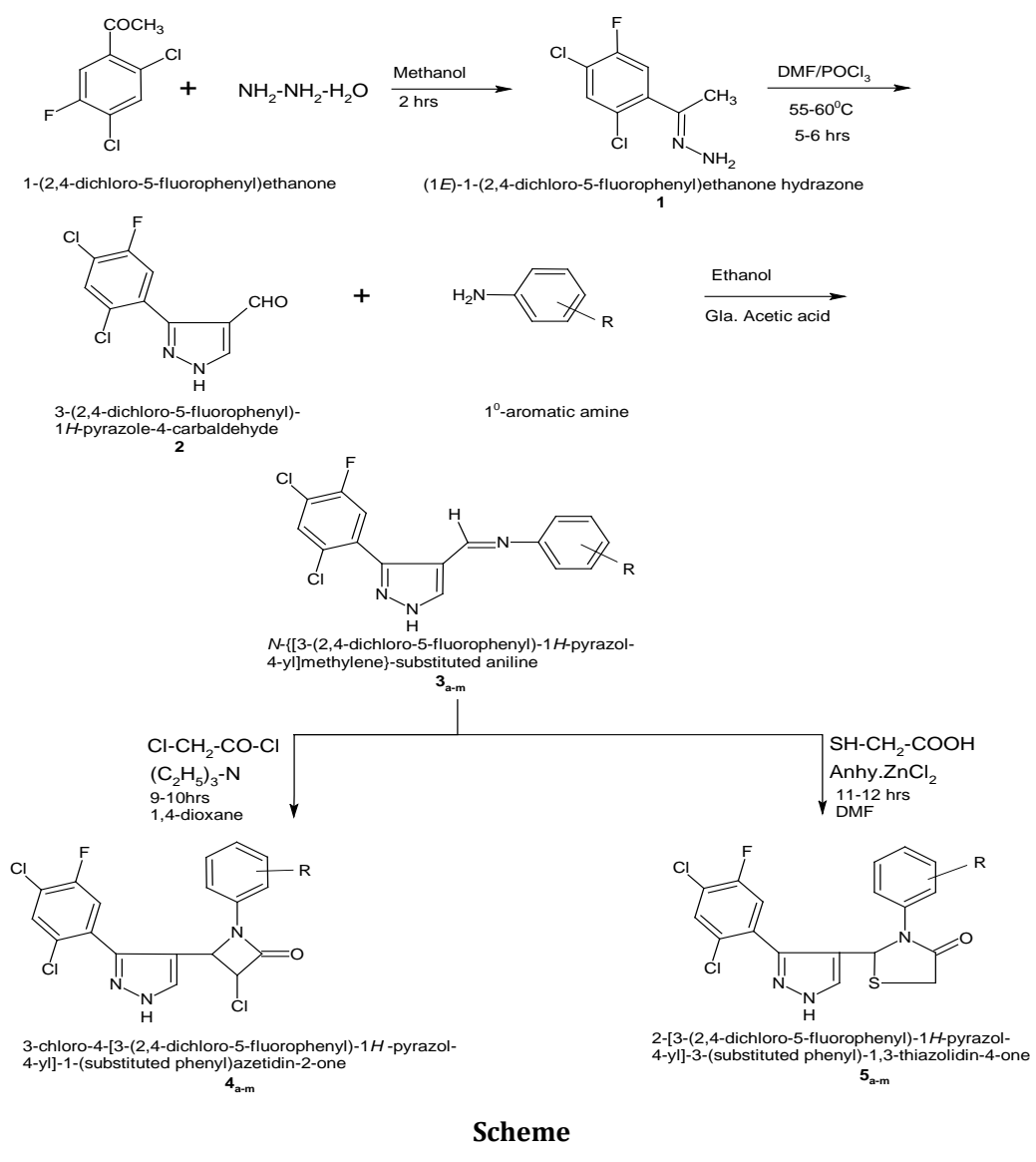


Table 1: Physical, characterization data of compound (3a-n), (4a-n) and (5a-n)

\begin{tabular}{|c|c|c|c|c|c|}
\hline S. No. & $\mathbf{R}$ & Mol. formula & Mol. Wt. gm/mol & M. P. ${ }^{\circ} \mathrm{C}$ & Yield \% \\
\hline $3 a$ & $\mathrm{H}$ & $\mathrm{C}_{16} \mathrm{H}_{10} \mathrm{~N}_{3} \mathrm{Cl}_{2} \mathrm{~F}$ & 334 & 120 & 68 \\
\hline $3 b$ & $2-\mathrm{Cl}$ & $\mathrm{C}_{16} \mathrm{H}_{9} \mathrm{~N}_{3} \mathrm{Cl}_{3} \mathrm{~F}$ & 368.5 & 140 & 64 \\
\hline $3 c$ & $3-\mathrm{Cl}$ & $\mathrm{C}_{16} \mathrm{H}_{9} \mathrm{~N}_{3} \mathrm{Cl}_{3} \mathrm{~F}$ & 368.5 & 120 & 62 \\
\hline $3 d$ & $4-\mathrm{Cl}$ & $\mathrm{C}_{16} \mathrm{H}_{9} \mathrm{~N}_{3} \mathrm{Cl}_{3} \mathrm{~F}$ & 368.5 & 156 & 65 \\
\hline $3 e$ & $2-\mathrm{NO}_{2}$ & $\mathrm{C}_{16} \mathrm{H}_{9} \mathrm{O}_{2} \mathrm{~N}_{4} \mathrm{Cl}_{2} \mathrm{~F}$ & 379 & 152 & 54 \\
\hline $3 f$ & $3-\mathrm{NO}_{2}$ & $\mathrm{C}_{16} \mathrm{H}_{9} \mathrm{O}_{2} \mathrm{~N}_{4} \mathrm{Cl}_{2} \mathrm{~F}$ & 379 & 140 & 50 \\
\hline $3 g$ & $4-\mathrm{NO}_{2}$ & $\mathrm{C}_{16} \mathrm{H}_{9} \mathrm{O}_{2} \mathrm{~N}_{4} \mathrm{Cl}_{2} \mathrm{~F}$ & 379 & 148 & 56 \\
\hline $3 \mathrm{~h}$ & $2-\mathrm{CH}_{3}$ & $\mathrm{C}_{17} \mathrm{H}_{12} \mathrm{~N}_{3} \mathrm{Cl}_{2} \mathrm{~F}$ & 348 & 180 & 0 \\
\hline $3 \mathrm{i}$ & $3-\mathrm{CH}_{3}$ & $\mathrm{C}_{17} \mathrm{H}_{12} \mathrm{~N}_{3} \mathrm{Cl}_{2} \mathrm{~F}$ & 348 & 182 & 58 \\
\hline $3 \mathrm{j}$ & $4-\mathrm{CH}_{3}$ & $\mathrm{C}_{17} \mathrm{H}_{12} \mathrm{~N}_{3} \mathrm{Cl}_{2} \mathrm{~F}$ & 348 & 180 & 60 \\
\hline $3 \mathrm{k}$ & $2-\mathrm{OCH}_{3}$ & $\mathrm{C}_{17} \mathrm{H}_{12} \mathrm{ON}_{3} \mathrm{Cl}_{2} \mathrm{~F}$ & 364 & 210 & 66 \\
\hline 31 & $3-\mathrm{OCH}_{3}$ & $\mathrm{C}_{17} \mathrm{H}_{12} \mathrm{ON}_{3} \mathrm{Cl}_{2} \mathrm{~F}$ & 364 & 190 & 60 \\
\hline $3 m$ & $4-\mathrm{OCH}_{3}$ & $\mathrm{C}_{17} \mathrm{H}_{12} \mathrm{ON}_{3} \mathrm{Cl}_{2} \mathrm{~F}$ & 364 & 204 & 64 \\
\hline $3 n$ & $\mathrm{C}_{10} \mathrm{H}_{7}$ (naphthyl) & $\mathrm{C}_{20} \mathrm{H}_{12} \mathrm{~N}_{3} \mathrm{Cl}_{2} \mathrm{~F}$ & 384 & 180 & 56 \\
\hline $4 a$ & $\mathrm{H}$ & $\mathrm{C}_{18} \mathrm{H}_{11} \mathrm{ON}_{3} \mathrm{Cl}_{3} \mathrm{~F}$ & 410.5 & 130 & 58 \\
\hline $4 b$ & $2-\mathrm{Cl}$ & $\mathrm{C}_{18} \mathrm{H}_{10} \mathrm{ON}_{3} \mathrm{Cl}_{4} \mathrm{~F}$ & 445 & 170 & 54 \\
\hline $4 c$ & $3-\mathrm{Cl}$ & $\mathrm{C}_{18} \mathrm{H}_{10} \mathrm{ON}_{3} \mathrm{Cl}_{4} \mathrm{~F}$ & 445 & 186 & 51 \\
\hline $4 d$ & $4-\mathrm{Cl}$ & $\mathrm{C}_{18} \mathrm{H}_{10} \mathrm{ON}_{3} \mathrm{Cl}_{4} \mathrm{~F}$ & 445 & 200 & 5 \\
\hline $4 \mathrm{e}$ & $2-\mathrm{NO}_{2}$ & $\mathrm{C}_{18} \mathrm{H}_{10} \mathrm{O}_{3} \mathrm{~N}_{4} \mathrm{Cl}_{3} \mathrm{~F}$ & 455.5 & 160 & 47 \\
\hline $4 f$ & $3-\mathrm{NO}_{2}$ & $\mathrm{C}_{18} \mathrm{H}_{10} \mathrm{O}_{3} \mathrm{~N}_{4} \mathrm{Cl}_{3} \mathrm{~F}$ & 455.5 & 120 & 46 \\
\hline $4 \mathrm{~g}$ & $4-\mathrm{NO}_{2}$ & $\mathrm{C}_{18} \mathrm{H}_{10} \mathrm{O}_{3} \mathrm{~N}_{4} \mathrm{Cl}_{3} \mathrm{~F}$ & 455.5 & 166 & 49 \\
\hline $4 \mathrm{~h}$ & $2-\mathrm{CH}_{3}$ & $\mathrm{C}_{19} \mathrm{H}_{13} \mathrm{ON}_{3} \mathrm{Cl}_{3} \mathrm{~F}$ & 424.5 & 196 & 54 \\
\hline $4 \mathrm{i}$ & $3-\mathrm{CH}_{3}$ & $\mathrm{C}_{19} \mathrm{H}_{13} \mathrm{ON}_{3} \mathrm{Cl}_{3} \mathrm{~F}$ & 424.5 & 132 & 42 \\
\hline $4 j$ & $4-\mathrm{CH}_{3}$ & $\mathrm{C}_{19} \mathrm{H}_{13} \mathrm{ON}_{3} \mathrm{Cl}_{3} \mathrm{~F}$ & 424.5 & 100 & 51 \\
\hline $4 \mathrm{k}$ & $2-\mathrm{OCH}_{3}$ & $\mathrm{C}_{19} \mathrm{H}_{13} \mathrm{O}_{2} \mathrm{~N}_{3} \mathrm{Cl}_{3} \mathrm{~F}$ & 440.5 & 220 & 44 \\
\hline 41 & $3-\mathrm{OCH}_{3}$ & $\mathrm{C}_{19} \mathrm{H}_{13} \mathrm{O}_{2} \mathrm{~N}_{3} \mathrm{Cl}_{3} \mathrm{~F}$ & 440.5 & 202 & 40 \\
\hline $4 \mathrm{~m}$ & $4-\mathrm{OCH}_{3}$ & $\mathrm{C}_{19} \mathrm{H}_{13} \mathrm{O}_{2} \mathrm{~N}_{3} \mathrm{Cl}_{3} \mathrm{~F}$ & 440.5 & 176 & 42 \\
\hline $4 n$ & $\mathrm{C}_{10} \mathrm{H}_{7}$ (naphthyl) & $\mathrm{C}_{22} \mathrm{H}_{13} \mathrm{ON}_{3} \mathrm{Cl}_{3} \mathrm{~F}$ & 460.5 & 190 & 40 \\
\hline $5 a$ & $\mathrm{H}$ & $\mathrm{C}_{18} \mathrm{H}_{12} \mathrm{ON}_{3} \mathrm{Cl}_{2} \mathrm{FS}$ & 408 & 127 & 56 \\
\hline $5 b$ & $2-\mathrm{Cl}$ & $\mathrm{C}_{18} \mathrm{H}_{11} \mathrm{ON}_{3} \mathrm{Cl}_{3} \mathrm{FS}$ & 442.5 & 152 & 48 \\
\hline $5 c$ & $3-\mathrm{Cl}$ & $\mathrm{C}_{18} \mathrm{H}_{11} \mathrm{ON}_{3} \mathrm{Cl}_{3} \mathrm{FS}$ & 442.5 & 173 & 47 \\
\hline $5 d$ & $4-\mathrm{Cl}$ & $\mathrm{C}_{18} \mathrm{H}_{11} \mathrm{ON}_{3} \mathrm{Cl}_{3} \mathrm{FS}$ & 442.5 & 200 & 50 \\
\hline $5 e$ & $2-\mathrm{NO}_{2}$ & $\mathrm{C}_{18} \mathrm{H}_{11} \mathrm{O}_{3} \mathrm{~N}_{4} \mathrm{Cl}_{2} \mathrm{FS}$ & 453 & 160 & 51 \\
\hline $5 f$ & $3-\mathrm{NO}_{2}$ & $\mathrm{C}_{18} \mathrm{H}_{11} \mathrm{O}_{3} \mathrm{~N}_{4} \mathrm{Cl}_{2} \mathrm{FS}$ & 453 & 120 & 48 \\
\hline $5 g$ & $4-\mathrm{NO}_{2}$ & $\mathrm{C}_{18} \mathrm{H}_{11} \mathrm{O}_{3} \mathrm{~N}_{4} \mathrm{Cl}_{2} \mathrm{FS}$ & 453 & 186 & 50 \\
\hline $5 h$ & $2-\mathrm{CH}_{3}$ & $\mathrm{C}_{19} \mathrm{H}_{14} \mathrm{ON}_{3} \mathrm{Cl}_{2} \mathrm{FS}$ & 422 & 120 & 58 \\
\hline $5 i$ & $3-\mathrm{CH}_{3}$ & $\mathrm{C}_{19} \mathrm{H}_{14} \mathrm{ON}_{3} \mathrm{Cl}_{2} \mathrm{FS}$ & 422 & 142 & 56 \\
\hline $5 j$ & $4-\mathrm{CH}_{3}$ & $\mathrm{C}_{19} \mathrm{H}_{14} \mathrm{ON}_{3} \mathrm{Cl}_{2} \mathrm{FS}$ & 422 & 168 & 60 \\
\hline $5 \mathrm{k}$ & $2-\mathrm{OCH}_{3}$ & $\mathrm{C}_{19} \mathrm{H}_{14} \mathrm{O}_{2} \mathrm{~N}_{3} \mathrm{Cl}_{2} \mathrm{FS}$ & 438 & 260 & 52 \\
\hline 51 & $3-\mathrm{OCH}_{3}$ & $\mathrm{C}_{19} \mathrm{H}_{14} \mathrm{O}_{2} \mathrm{~N}_{3} \mathrm{Cl}_{2} \mathrm{FS}$ & 438 & 210 & 50 \\
\hline $5 m$ & $4-\mathrm{OCH}_{3}$ & $\mathrm{C}_{19} \mathrm{H}_{14} \mathrm{O}_{2} \mathrm{~N}_{3} \mathrm{Cl}_{2} \mathrm{FS}$ & 438 & 224 & 52 \\
\hline $5 n$ & $\mathrm{C}_{10} \mathrm{H}_{7}$ (naphthyl) & $\mathrm{C}_{22} \mathrm{H}_{14} \mathrm{ON}_{3} \mathrm{Cl}_{2} \mathrm{FS}$ & 458 & 230 & 42 \\
\hline
\end{tabular}

\section{Antimicrobial activity}

Following common standard strains were used for screening of antibacterial and antifungal activities: E. Coli (MTCC 442), $P$. Aeruginosa (MTCC 441), S. Aureus (MTCC 96), S. Pyogenus (MTCC 443), C. Albicans (MTCC 227), A. Niger (MTCC 282), A. Clavatus (MTCC 1323). The strains were procured from Institute of Microbial Technology, Chandigarh. DMSO was used as diluents/vehicle to get desired concentration of drugs to test upon standard bacterial strains. Each synthesised drug was diluted for obtaining 2000 microgram $/ \mathrm{ml}$ concentration, as a stock solution. In primary screening 1000 microgram $/ \mathrm{ml}, 500$ microgram $/ \mathrm{ml}$, and 250 microgram $/ \mathrm{ml}$ concentrations of the synthesised drugs were taken. The actively synthesised drugs found in this primary screening were further tested in the second set of dilution against all microorganisms. The drugs found active in primary screening were similarly diluted to obtain 200 microgram $/ \mathrm{ml}, 100$ microgram $/ \mathrm{ml}$,
50 microgram $/ \mathrm{ml}, 25$ microgram $/ \mathrm{ml}, 12.5$ microgram $/ \mathrm{ml}$ and 6.250 microgram $/ \mathrm{ml}$ concentrations. The highest dilution showing at least $99 \%$ inhibition zone is taken as MIC. The result of this is much affected by the size of the inoculums. Gentamycin, Ampicillin, Chloramphenicol, Ciprofloxacin, Norfloxacin, Nystatin and Griseofulvin were used as a standard. The Comparative activities of the newly synthesised compounds and the control antibiotics on bacterial and fungal strains respectively were summarised in table 2 and table 3.

Excellent to good activity was observed in compounds $4 \mathrm{~d}$ (against $E$ Coli, P. Aeruginosa, S. Aureus, S. Pyogenus), compounds $3 \mathrm{~g}, 4 \mathrm{e}, 5 \mathrm{~g}, 5 \mathrm{n}$ (against E. Coli, S. Aureus, S. Pyogenus), compounds 3a, 3b, 3j, 3l, 4j, 4l, $4 \mathrm{~m}, 5 \mathrm{e}, 5 \mathrm{~h}$ (against E. Coli, S. Aureus) as well as compounds 3a, 3c, 3f, 3g, 3h, 3j, 3k, 3l, 4c, 4e, 4f, 4g, 4h, 4k, 4l, 4m, 5b, 5e, 5f, 5g, 5i, 5j, 5l, 5m (against $C$. Albicans). The remaining compounds were found effective at a much higher concentration as compared to the standard drugs.

Table 2: Antibacterial activity of compounds 3a-n, 4a-n and 5a-n

\begin{tabular}{|c|c|c|c|c|}
\hline Code & E. Coli & P. Aeruginosa & S. Aureus & S. Pyogenus \\
\hline No. & MTCC 442 & MTCC 441 & MTCC 96 & MTCC 443 \\
\hline $3 a$ & 100 & 200 & 250 & 125 \\
\hline $3 b$ & 62.5 & 100 & 125 & 200 \\
\hline $3 c$ & 200 & 125 & 250 & 62.5 \\
\hline $3 d$ & 250 & 200 & 200 & 200 \\
\hline
\end{tabular}




\begin{tabular}{|c|c|c|c|c|}
\hline $3 e$ & 200 & 125 & 250 & 125 \\
\hline $3 \mathrm{f}$ & 125 & 125 & 250 & 62.5 \\
\hline $3 g$ & 62.5 & 200 & 100 & 100 \\
\hline $3 \mathrm{~h}$ & 200 & 250 & 250 & 250 \\
\hline $3 \mathrm{i}$ & 250 & 250 & 500 & 100 \\
\hline $3 j$ & 100 & 200 & 250 & 125 \\
\hline $3 \mathrm{k}$ & 250 & 200 & 100 & 125 \\
\hline 31 & 100 & 125 & 100 & 250 \\
\hline $3 \mathrm{~m}$ & 62.5 & 250 & 500 & 500 \\
\hline $3 n$ & 200 & 125 & 250 & 500 \\
\hline $4 a$ & 200 & 250 & 500 & 500 \\
\hline $4 b$ & 100 & 200 & 500 & 250 \\
\hline $4 c$ & 125 & 100 & 250 & 500 \\
\hline $4 d$ & 62.5 & 50 & 100 & 100 \\
\hline $4 \mathrm{e}$ & 100 & 125 & 250 & 100 \\
\hline $4 \mathrm{f}$ & 250 & 100 & 200 & 200 \\
\hline $4 g$ & 500 & 500 & 100 & 200 \\
\hline $4 h$ & 250 & 250 & 500 & 500 \\
\hline $4 i$ & 200 & 200 & 200 & 250 \\
\hline $4 j$ & 62.5 & 100 & 200 & 250 \\
\hline $4 \mathrm{k}$ & 200 & 250 & 250 & 250 \\
\hline 41 & 100 & 62.5 & 62.5 & 125 \\
\hline $4 \mathrm{~m}$ & 62.5 & 100 & 200 & 200 \\
\hline $4 n$ & 125 & 100 & 250 & 250 \\
\hline $5 a$ & 200 & 125 & 100 & 125 \\
\hline $5 b$ & 125 & 62.5 & 100 & 100 \\
\hline $5 c$ & 200 & 62.5 & 100 & 250 \\
\hline $5 d$ & 200 & 250 & 250 & 250 \\
\hline $5 e$ & 100 & 125 & 250 & 500 \\
\hline $5 \mathrm{f}$ & 200 & 250 & 200 & 250 \\
\hline $5 g$ & 62.5 & 100 & 100 & 62.5 \\
\hline $5 \mathrm{~h}$ & 62.5 & 125 & 250 & 250 \\
\hline $5 i$ & 250 & 250 & 250 & 500 \\
\hline $5 j$ & 100 & 125 & 500 & 500 \\
\hline $5 \mathrm{k}$ & 250 & 250 & 125 & 100 \\
\hline 51 & 250 & 500 & 500 & 500 \\
\hline $5 \mathrm{~m}$ & 500 & 250 & 500 & 500 \\
\hline $5 n$ & 100 & 125 & 250 & 100 \\
\hline Ampicillin & 100 & -- & 250 & 100 \\
\hline Chloramphenicol & 50 & 50 & 50 & 50 \\
\hline Ciprofloxacin & 25 & 25 & 50 & 50 \\
\hline Norfloxacin & 10 & 10 & 10 & 10 \\
\hline
\end{tabular}

Table 3: Antifungal activity of compounds 3a-n, 4a-n and 5a-n

\begin{tabular}{|c|c|c|c|}
\hline Code & C. albicans & A. niger & A. clavatus \\
\hline No. & MTCC 227 & MTCC 282 & MTCC 1323 \\
\hline $3 a$ & 500 & 250 & 250 \\
\hline $3 b$ & 1000 & 1000 & $>1000$ \\
\hline $3 c$ & 250 & 1000 & 1000 \\
\hline $3 d$ & $>1000$ & 1000 & 1000 \\
\hline $3 e$ & $>1000$ & 1000 & 1000 \\
\hline $3 f$ & 500 & 500 & 500 \\
\hline $3 g$ & 500 & 500 & 1000 \\
\hline $3 \mathrm{~h}$ & 250 & 500 & 500 \\
\hline $3 \mathrm{i}$ & $>1000$ & 1000 & 1000 \\
\hline $3 j$ & 100 & $>1000$ & $>1000$ \\
\hline $3 \mathrm{k}$ & 500 & 1000 & 1000 \\
\hline 31 & 500 & $>1000$ & $>1000$ \\
\hline $3 \mathrm{~m}$ & 1000 & 500 & 500 \\
\hline $3 n$ & $>1000$ & 500 & 500 \\
\hline $4 a$ & $>1000$ & 500 & 500 \\
\hline $4 \mathrm{~b}$ & $>1000$ & 500 & 500 \\
\hline $4 c$ & 500 & 1000 & 1000 \\
\hline $4 d$ & 1000 & 1000 & 500 \\
\hline $4 \mathrm{e}$ & 500 & $>1000$ & $>1000$ \\
\hline $4 \mathrm{f}$ & 500 & 200 & 500 \\
\hline $4 g$ & 250 & 1000 & 1000 \\
\hline $4 \mathrm{~h}$ & 500 & 1000 & 1000 \\
\hline $4 i$ & 1000 & 500 & 500 \\
\hline $4 j$ & 1000 & 1000 & 1000 \\
\hline $4 \mathrm{k}$ & 250 & 1000 & $>1000$ \\
\hline 41 & 500 & 500 & 1000 \\
\hline
\end{tabular}




\begin{tabular}{llll}
\hline $4 \mathrm{~m}$ & 500 & 250 & 500 \\
$4 \mathrm{n}$ & 1000 & $>1000$ & $>1000$ \\
$5 \mathrm{a}$ & 1000 & 500 & 500 \\
$5 \mathrm{~b}$ & 500 & 500 & 500 \\
$5 \mathrm{c}$ & 1000 & 1000 & $>1000$ \\
$5 \mathrm{~d}$ & $>1000$ & $>1000$ & 500 \\
$5 \mathrm{e}$ & 500 & 500 & 500 \\
$5 \mathrm{f}$ & 250 & 500 & $>1000$ \\
$5 \mathrm{~g}$ & 500 & $>1000$ & $>1000$ \\
$5 \mathrm{~h}$ & 1000 & 500 & $>1000$ \\
$5 \mathrm{i}$ & 250 & $>1000$ & $>1000$ \\
$5 \mathrm{j}$ & 250 & 1000 & $>1000$ \\
$5 \mathrm{k}$ & 1000 & $>1000$ & 500 \\
$5 \mathrm{l}$ & 500 & 500 & 1000 \\
$5 \mathrm{~m}$ & 200 & 500 & 100 \\
$5 \mathrm{n}$ & $>1000$ & 500 & 100 \\
Nystatin & 100 & 100 & \\
Greseofulvin & 500 & 100 & \\
\hline
\end{tabular}

\section{RESULTS AND DISCUSSION}

\section{The compounds were synthesised as per scheme}

Compound (1E)-1-(2,4-Dichloro-5-fluorophenyl) ethanone hydrazone 1 were synthesized from 1-(2,4-dichloro-5-fluorophenyl) ethanone, which upon reaction with DMF/POCl3 yields 3-(2,4-dichloro-5-fluoro phenyl)1H-pyrazol-4-carbaldehyde 2. Compounds N-\{[3-(2,4-dichloro-5-fluoro phenyl)-1H-pyraol-4-yl] methylene $\}$ substituted anilin [3a-n] were synthesized from 3-(2,4-dichloro-5-fluoro phenyl)-1H-pyrazol-4carbaldehyde 2 and various aromatic amine, which upon cyclization with chloro acetyl chloride and thioglycolic acid yields 3-Chloro-4-[3(2,4-dichloro-5-fluoro phenyl)-1H-pyrazol-4-yl]-1-(substituted) azetidin -2-one [4a-n] and 2-[3-(2,4-Dichloro-5-fluoro phenyl)-1H-pyraol-4-yl]-3(substituted phenyl)-1,3-thiazolidin-4-one [5a-n] respectively. The proposed structures of all the synthesised compounds were well supported by IR, $1 \mathrm{H} \mathrm{NMR},{ }^{13} \mathrm{C}$ NMR and mass spectral data. The formation of compounds 3a-n was confirmed by the appearance of singlet signal at $\delta$ 9.747-9.750 for $\mathrm{CH}=\mathrm{N}$ system. The $1 \mathrm{H}$ NMR spectrum also displayed signals at $\delta$ 5.103-5.105 for $\mathrm{CH}-\mathrm{N}$ of azetidine ring and at $\delta$ 4.01-3.975 for $\mathrm{CH} 2-\mathrm{S}$ of thiazolidinone ring system respectively. Aromatic protons were observed in the usual region as multiplet between $\delta 7.328-7.535, \delta 7.305-8.049, \delta 7.323-7.703$.

\section{CONCLUSION}

In summary, $\mathrm{N}-\{[3-(2,4-$ dichloro-5-fluoro phenyl)-1H-pyraol-4-yl] methylene \} substituted anilin [3a-n], 3-Chloro-4-[3-(2,4-dichloro-5fluoro phenyl)-1H-pyrazol-4-yl]-1-(substituted) azetidin-2-one [4a-n] and 2-[3-(2,4-Dichloro-5-fluoro phenyl)-1H-pyraol-4-yl]-3-(substituted phenyl)-1,3-thiazolidin-4-one [5a-n] derivatives have been synthesized and characterized. In vitro antimicrobial testing of the compounds was carried out by microdilution Method. Amongst the synthesised compounds, many of them had proven their antimicrobial potency which varies from good to excellent. Excellent to good activity was observed in compounds 4d (against E. Coli, P. Aeruginosa, S. Aureus, $S$. Pyogenus), compounds 3g, 4e, 5g, 5n (against E. Coli, S. Aureus, $S$. Pyogenus), compounds 3a, 3b, 3j, 3l, 4j, 4l, 4m, 5e, 5h (against E. Coli, $S$. Aureus) as well as compounds 3a, 3c, 3f, 3g, 3h, 3j, 3k, 3l, 4c, 4e, 4f, 4g, 4h, $4 \mathrm{k}, 4 \mathrm{l}, 4 \mathrm{~m}, 5 \mathrm{~b}, 5 \mathrm{e}, 5 \mathrm{f}, 5 \mathrm{~g}, 5 \mathrm{i}, 5 \mathrm{j}, 5 \mathrm{l}, 5 \mathrm{~m}$ (against $C$. Albicans). The remaining compounds were found effective at a much higher concentration as compared to the standard drugs.

\section{ACKNOWLEDGEMENT}

The services of SAIF, CDRI, Lucknow and Micro care Lab Surat is acknowledged for spectral analysis and antimicrobial testing respectively.

\section{CONFLICT OF INTERESTS}

\section{Declared none}

\section{REFERENCES}

1. Akhaja T. 1,3-dihydro-2H-indol-2-ones derivatives: Design, Synthesis, in vitro antibacterial, antifungal and antitubercular study. Eur J Med Chem 2011;46:5573-9.
2. Pandey V, Chawla V, Saraf S. Comparative study of the conventional and microwave-assisted synthesis of some Schiff bases and their potential as antimicrobial agents. Med Chem Res 2011;21:4500-12.

3. Masunari A. A new class of nifuroxazide analogues: synthesis of 5-nitrophene derivatives with antimicrobial activity against multidrug-resistant Staphylococcus aureus. Bioorg Med Chem 2007;15:4229-36.

4. Chavan AA, Pai NR. Synthesis and biological activity of NSubstituted-3-chloro-2-azetidinones. Molecules 2007;12:2467-77.

5. Kumar A, Rajput CS. Synthesis and anti-inflammatory activity of newer quinazolin-4-one derivatives. Eur J Med Chem 2009;44:83-90.

6. Leach CA, Deirdre MB. Lipoprotein-associated PLA2 inhibitiona novel, non-lipid lowering strategy for atherosclerosis therapy. II Farmaco 2001;56:45-50.

7. Banik BK, Banik I, Becker FF. Stereocontrolled synthesis of anticancer $\beta$-lactams via the Staudinger reaction. Bioorg Med Chem 2005;13:3611-22.

8. Patel KH, Mehta AG. Synthesis and antifungal activity of azetidines and thiazolidinediones derivative of 2-amino-6-(2naphthalenyl) thiazolo $[3,2-d]$ thiadiazole. E J Chem 2006;3:267-73.

9. Veinberg G, Shestakova I, Vorona M, Kanepe I, Lukevics E. Synthesis of antitumor 6-alkylidenepenicillanate sulfones and related 3-alkylidene-2-azetidinones. Bioorg Med Chem Lett 2004; $14: 147-50$.

10. Narute AS, Khedekar PB, Bhusari KP. QSAR studies on 4thiazolidinoes and 2-azetidinones bearing benzothiophene nucleus as potential anti-tubercular agents. Indian J Chem 2008;47B:586-91.

11. Datta NJ, Khunt RC, Parikh AR. Synthesis of some new 4thiazolidinones as biologically potent agents. Indian J Chem 2002;41B:433-5.

12. Patel KD, Mistry BD, Desai KR. Synthesis and biological screening of 2-thiazolidinones and 4-thiazolidinones. J Indian Chem Soc 2004;81:783-5.

13. Yadav R, Srivastava SD, Srivastava SK. Synthesis, antimicrobial and anti-inflammatory activities of 4-oxothiazolidines and their 5-arylidines. Indian J Chem 2005;44B:1262-6.

14. Mistry K, Desai KR. Microwave assisted rapid and efficient synthesis of nitrogen and sulphur containing heterocyclic compounds and their pharmacological evaluation. Indian J Chem 2006;45(B):1762-6.

15. Gurupadyya BM, Gopal M, Padmashali B, Manohara YN. Synthesis and pharmacological evaluation of azetidin-2-ones and thiazolidin-4-ones encompassing benzothiazole. Indian J Pharm Sci 2008;70:572-7.

16. Milan C, Maja M, Nela D. Design and synthesis of some thiazolidin-4-ones based on (7-Hydroxy-2-oxo-2H-chromen-4yl) acetic acid. Molecule 2009;14:2501-13.

17. Omar K, Geronikaki A, Zoumpoulakis P, Camoutsis C, Sokovic M, Ciric A, et al. Novel 4-thiazolidinone derivatives as potential 
antifungal and antibacterial drugs. Bioorg Med Chem 2010;18:426-32.

18. Goel N, Drabu S, Bawa S. Antimicrobial screening and one-pot synthesis of 4-(substituted-aminomethyl)-3-(2-naphthyl)-1-phenyl1H-pyrazole derivatives. J Pharm Bioallied Sci 2014;6:253-9.

\section{How to cite this article}

- $\quad$ Bhavnaben D Mistry, Kishor R Desai, Nigam J Desai. Synthesis, in vitro antimicrobial activity of schiff's base, azetidinones and thiazolidinones. Int J Curr Pharm Res 2017;9(1):126-131. 